\title{
«Back to the roots» oder eine Alternative zu den Begriffen Schulmedizin und CAM
}

\author{
Eugen Judin \\ Düsseldorf, Deutschland
}

Eine stichhaltige Klassifizierung der uns umgebenden Realität - der Objekte, der Krankheitssymptome, der Gefühle usw. - stellt die Krönung eines Prozesses der Erkenntnisbildung dar. Eine Vernachlässigung der etablierten Klassifizierungsprinzipien führt durch Vermischung von fremdartigen Begriffen $\mathrm{zu}$ terminologischem Chaos und mündet folgerichtig in falschen, in Bezug auf die Medizin potenziell gesundheitsschädigenden Handlungen. Unscharfe Begrifflichkeiten beeinflussen Gedankengänge und Handlungen der Ärzte, führen einerseits zur destruktiven Vereinfachung, andererseits zur unnötigen Verkomplizierung der Behandlungsabläufe. Die Bedeutung einer adäquaten Klassifizierung, Bergriffsbildung und Begriffsbenutzung wurde auch innerhalb dieser Zeitschrift bereits thematisiert [1-3].

Mediziner sind in den letzen Jahrzehnten mit einer Vielzahl von neu entwickelten Begriffen, deren Bedeutung nicht immer klar definiert ist, konfrontiert: Schulmedizin (Conventional Medicine), Evidence Based Medicine, Complementary and Alternative Medicine (CAM), Integrative Medicine, Traditional Chinese Medicine (TCM), Person-Centered Medicine, Biomedizin, biopsychosoziale Medizin usw.

So wird aktuelle Medizin häufig in Schulmedizin bzw. CAM klassifiziert.

Dabei sollte eigentlich grundsätzlich gelten: Wenn irgendetwas als alternativ oder komplementär benannt wird, dann muss als Erstes klar definiert werden, was man unter dem Referenzbegriff, in unserem Fall der Schulmedizin, versteht. Bis jetzt fehlt es aber an einer stichhaltigen Definition der Schulmedizin. Unter dem Begriff CAM werden zudem oft Methoden zusammengefasst, die miteinander gar nichts gemeinsam haben. Dazu kommt in regelmäßiger Folge die praktische Erkenntnis, dass das, was man heute als schulmedizinisch und anerkannt definiert, morgen schon als obsolet und gesundheitsschädigend eingestuft wird. Es scheint also so zu sein, als wäre es nicht möglich, mit einer ausreichenden Deutlichkeit die Begriffe Schulmedizin und CAM zu definieren. Allerdings gibt es in der Medizin auch hervorragende Begriffe, die leider in die Vergessenheit geraten sind und in der aktuellen Literatur fast gar nicht angewendet werden: z.B. ätiopathogenetische Therapie bzw. Medizin.

Eine Therapie wirkt entweder auf die Ursache der Erkrankung oder auf die Mechanismen der Erkrankungsentwicklung oder auf beides. Es ist unbestreitbar, dass die ganze medizinisch-wissenschaftliche Forschung in erster Linie darauf gerichtet werden soll, die Ursachen und Entwicklungsmechanismen der Erkrankungen aufzudecken. Nur so lassen sich Therapien entwickeln, die auf die Erkrankung zugeschnitten sind. Es muss erwähnt werden, dass das ätiopathogenetische Herangehen zur Erarbeitung eines Behandlungskonzepts in krassem Gegensatz zum evidenzbasierten Herangehen steht. Letzteres setzt vor allem auf eine vergleichende Überprüfung der Therapien mittels statistischer Verfahren und ignoriert die Ätiopathogenese der Erkrankung vollkommen.

Eine ätiopathogenetisch gerichtete Medizin ist allerdings nicht mit dem sogenannten biomedizinischen Modell (biomedical model) gleichzusetzen. Der Terminus biomedizinisches Modell ist mit einer negativen Schattierung behaftet und signalisiert, dass man bei Erkrankungen mit bekannter Ätiologie eingeschränkt und mechanistisch denkt und vorgeht. So entfernt man strikt nach der Ätiologie und ohne Notwendigkeit beispielsweise einen Discus intervertebralis, der für einen Schmerzsyndrom ätiologisch verantwortlich ist. Wenn man aber die Pathogenese eines vertebragenen Schmerzsyndroms (inklusive der psychologisch-neurophysiologischen Schmerzkomponente) kennt, weiß man, dass sich in vielen Fällen die Bandscheibenschäden und Schmerzen unter einer pathogenetisch adäquat ausgerichteten Therapie auch ohne Operation völlig zurückbilden können. Das heißt, im Optimalfall sollten alle Feinheiten der Ätiopathogenese bei

\section{KARGER \\ Fax +497614520714 \\ Information@Karger.de}

www.karger.com (c) 2011 S. Karger GmbH, Freiburg

1661-4119/11/0182-0106 \$38.00/0

Accessible online at:

www.karger.com/fok
Eugen Judin

Lindemannstraße 5

40237 Düsseldorf, Deutschland

eugenjudin@gmx.de 
der Gestaltung der Therapiemaßnahmen berücksichtigt werden. Das nennt man - bezogen auf das klinische Bild eines individuellen Patienten - das klinische Denken, ein Begriff, der genauso wie der Begriff ätiopathogenetische Therapie durch den Einfluss der evidenzbasierten Medizin und einer Depersonalisierung der Medizin aus dem Sprachgebrauch fast verschwunden ist.

Nach meiner Ansicht sollten die Therapiemethoden in solche mit klarem und in solche mit unklarem ätiopathogenetischen Prinzip aufgeteilt werden. Die aus Erfahrung wirksamen Therapien mit unklarem Wirkprinzip bei einer ätiopathogenetisch erforschten Erkrankung oder die gut erforschten Therapien, die bei einer Erkrankung mit unklarer Ätiopathogenese aus Erfahrung wirksam sind, sollen dann als solche definiert und zum Objekt der wissenschaftlichen Forschung werden. Das könnte den Wirkungskreis bilden, der heute der CAM zugeteilt wird. Diese Ausrichtung würde mehr Wissenschaftlichkeit, Prägnanz und Seriösität in die Struktur der CAM bringen.

Der wissenschaftliche Fokus sollte wieder auf die Grundlagenforschung, die Erforschung der Ätiopathogenese der Erkrankungen sowie auf eine Analyse von klinisch adäquat strukturierten Beobachtungsstudien gerichtet werden und damit weg von der Dominanz der statistischen Auslegungen der evidenzbasierten Medizin.

Sinnvoll wäre es in diesem Zusammenhang, die Medizin nicht in Schulmedizin und CAM aufzuteilen, sondern in eine klinisch adäquat applizierte ätiopathogenetische Medizin und in eine Medizin, die noch nach einem ätiopathogenetischen Anschluss sucht.

\section{Literatur}

1 Walach H: «Integrative Medizin» - die Kolonialisierung des Anderen und die Notwendigkeit des ganz Anderen. Forsch Komplementmed 2010;17: $4-6$
Walach H, Brinkhaus B, Heusser P, Reiter B, Michalsen A, Witt C, Lüdtke R: Complementary or integrative - ideas and notions. Forsch Komplementmed 2010;17:215-220.
Roberti di Sarsina P, Iseppato I: Person-centred medicine: towards a definition. Forsch Komplementmed 2010;17:277-278. 